\title{
Non-dipping blood pressure patterns and arterial stiffness parameters in patients with Behcet's disease
}

\author{
Gulperi Celik ${ }^{1}$, Sema Yilmaz ${ }^{2}$ and Serpil Ergulu Esmen ${ }^{2}$
}

Behcet's disease is a multisystemic vasculitis involving veins and arteries of various sizes. Non-dipping status, augmentation index and pulse wave velocity are important determinants of cardiovascular mortality and morbidity. We investigated the nondipping status and arterial stiffness in patients with Behcet's disease. In this cross-sectional study, we examined the vascular parameters of 96 patients with Behcet's disease (53\% female) and 60 age- and sex-matched control subjects. The non-dipping status and arterial distensibility were assessed using a Mobil-0-Graph Arteriograph, an automatic oscillometric device. In total, $65.6 \%$ of 96 patients were systolic non-dippers, and $34.4 \%$ exhibited high augmentation indices. Ten percent of the control subjects were systolic non-dippers, and $11.7 \%$ exhibited high augmentation indices. Nocturnal decreases in systolic blood pressure correlated with central systolic blood pressure and diastolic blood pressure, as well as nocturnal decreases in diastolic blood pressure. Furthermore, non-dipper patients with Behcet's disease exhibited higher nocturnal cardiac outputs than did dipper patients with Behcet's disease. Augmentation index correlated negatively with C-reactive protein and correlated positively with both $24 \mathrm{~h}$ and nocturnal peripheral resistance, as well as $24 \mathrm{~h}$ pulse wave velocity. The patients with high augmentation indices exhibited lower creatinine clearance, as well as lower nocturnal cardiac outputs, higher $24 \mathrm{~h}$ peripheral resistance and higher $24 \mathrm{~h}$ pulse wave velocities. Non-dipping status and arterial stiffness may exacerbate the harmful cardiovascular effects of the other. In addition to conventional risk factors, non-dipping status and arterial stiffness should be examined during the follow-up evaluations of patients with Behcet's disease.

Hypertension Research (2015) 38, 856-861; doi:10.1038/hr.2015.86; published online 13 August 2015

Keywords: augmentation index; Behcet's disease; cardiac output; non-dipping status; pulse wave velocity

\section{INTRODUCTION}

Behcet's disease (BD) was first described in 1937 by Hulusi Behcet and is an autoinflammatory disease and multisystemic vasculitis of an unknown origin characterized by mucocutaneous, ocular, arthritic and vascular manifestations. ${ }^{1-3}$ As the primary pathological feature of $\mathrm{BD}$ is vasculitis, it is often exacerbated by vascular complications, with an incidence of vascular involvement ranging from 7 to $29 \% .{ }^{4}$ Largevessel vasculitis occurs in a subgroup of patients with $\mathrm{BD}$ at high risk for disease-related morbidity and mortality. ${ }^{5}$ Recognition of patients at risk, as well as the early detection of vasculitis and aggressive treatment, is essential for providing optimal care for these patients. ${ }^{5,6}$

Twenty-four hour ambulatory blood pressure (BP) monitoring has been used to demonstrate the diurnal rhythm of arterial BP. ${ }^{7}$ After many decades of investigation, it has been established that target organ damage is more closely associated with ambulatory BP monitoring than with clinical BP, and specific features of the $24 \mathrm{~h}$ BP pattern have been linked to the progressive injury of target tissues and the triggering of both cardiac and cerebrovascular events. ${ }^{8-10}$ Among normotensive individuals, both the systolic BP (SBP) and the diastolic BP (DBP) of this so-called sleep-time dipper pattern exhibit an abrupt rise in the morning, just before the commencement of diurnal activity, peaking during the morning or afternoon hours. Thereafter, these pressures gradually decline, reaching their lowest levels during night-time sleep. This BP circadian pattern is characterized by night-time SBP and DBP mean levels 10-20\% lower than those observed during the daytime. In normotensive and hypertensive individuals, the failure of night-time $\mathrm{BP}$ to decline $>10 \%$ compared with daytime BP (non-dipping status) is associated with increased target organ damage. The circadian rhythm of BP also affects cardiovascular disease. ${ }^{11-13}$

Several authors have demonstrated that arterial stiffness is increased in BD. ${ }^{14-17}$ Ample data exist demonstrating that increased systemic inflammation has a deleterious effect on both the structure and the function of the arterial wall and may enhance the atherosclerotic process. ${ }^{14,16,18}$ Both acute and chronic inflammation have been linked with increased arterial stiffness and altered pressure wave reflections. ${ }^{14-17}$

Arterial stiffness is a reliable predictor of subsequent cardiovascular mortality and vascular damage. ${ }^{19}$ Augmentation index (AIx) and pulse wave velocity (PWV) are ideal indicators of arterial stiffness. ${ }^{2,15,17,20,21}$

${ }^{1}$ Division of Nephrology, Department Of Internal Medicine, Faculty of Medicine, Selcuk University, Konya, Turkey and ${ }^{2}$ Division of Rheumatology, Department of Internal Medicine, Faculty of Medicine, Selcuk University, Konya, Turkey

Correspondence: Dr G Celik, Division of Nephrology, Department of Internal Medicine, School of Medicine, Selcuk University, Konya 42075, Turkey E-mail: gulpericelik@gmail.com

Received 14 January 2015; revised 2 June 2015; accepted 8 June 2015; published online 13 August 2015 
In other words, PWV and AIx have been used to evaluate the viscoelastic properties of the large arteries. ${ }^{2,22}$ Increased and premature pressure reflections emerge that are superimposed over the generic pulse wave ejected by the heart. Their accumulation results in an elevation of aortic SBP known as augmentation. The percent ratio of this augmentation to the aortic pulse pressure is called AIx, which represents a percentage of the central pulse pressure. The AIx is often normalized against a heart rate of 75 beats $\min ^{-1} .23$

There are few data regarding the indicators of arterial stiffness, nondipping patterns and related parameters in these patients. In a cohort of 96 consecutive patients with $\mathrm{BD}$, we assessed dipping patterns in an attempt to interpret the different pathophysiological mechanisms underlying the potential alterations in both central and peripheral hemodynamics. We also examined the parameters related to the magnitude of wave reflection pressure and local large artery stiffness. We hypothesized that patients with $\mathrm{BD}$ exhibit high rates of nondipper BP patterns; however, these patients may also experience central and peripheral hemodynamic disturbances that adversely affect arterial stiffness parameters.

\section{MATERIALS AND METHODS}

\section{Subjects}

This study included 96 patients with BD who fulfilled the International Study Group criteria ${ }^{24}$ and control subjects. For the control group, 60 healthy ageand sex-matched subjects were recruited from our hospital staff and from among healthy volunteers. The study was approved by the Hospital Ethics Committee, and informed, written consent was provided by each patient. Patients were included if they met the following criteria: $>18$ years of age; an office BP <140/90 $\mathrm{mm} \mathrm{Hg}$; and a $24 \mathrm{~h} \mathrm{BP}<125 / 80 \mathrm{~mm} \mathrm{Hg}$. We took great care to exclude patients with known cardiovascular disease or risk factors for cardiovascular disease. The exclusion criteria included a major cardiovascular disease, including previous myocardial infarction, congestive heart failure, valvular heart disease, atrial fibrillation, arterial hypertension, insulin-dependent diabetes mellitus, non-insulin-dependent diabetes mellitus, dyslipidemia, excessive alcohol consumption and the use of antihypertensive medications and additional cardiovascular or metabolic drugs, including statins, hormone replacement therapy, nitrates, angiotensin-converting enzyme inhibitors, angiotensin receptor blockers and diuretics. Smoking habits were also taken into account. The duration of the disease in the $\mathrm{BD}$ group was calculated as the time at which the International Study Group criteria were fulfilled until the time of examination.

For each patient, the clinical data included age, sex, cutaneous (genital and oral) ulcers and eye, central nervous system, arterial, venous and joint involvement. Medical treatment included colchicine, corticosteroids (0.5-1 $\mathrm{mg} \mathrm{kg}^{-1}$ per day), immunosuppressants (cyclophosphamide or azathioprine), oral anticoagulation and low-dose aspirin. Each patient was evaluated during both active and inactive disease periods. At the time of examination, the presence of two or more of the following clinical features denoted active disease: oral ulceration; genital ulceration; skin lesions; ocular lesions; active major vessel disease; and active major organ involvement, including active gastrointestinal or neurological lesions. Subjects with either one lesion or no lesions had inactive disease.

\section{Biochemical data}

A venous blood sample was obtained from each patient following overnight fasting. Serum creatinine, urea, glucose, total cholesterol, triglyceride, highdensity lipoprotein cholesterol, low-density lipoprotein cholesterol, albumin, total protein, calcium, phosphorus, C-reactive protein (CRP), $24 \mathrm{~h}(\mathrm{~h})$ urinary uric acid, $24 \mathrm{~h}$ urinary albumin and $24 \mathrm{~h}$ urine protein were measured using an ARCHITECT C16000 (Abbott Diagnostics, Illinois, IL, USA). The erythrocyte sedimentation rate (ESR) was determined using a TEST-1 analyzer (Alifax, Padova, Italy). Complete blood count measurements, including hemoglobin, hematocrit and lymphocytes, were performed using a Sysmex XT 2000 (Roche, Basel, Switzerland).

\section{Hemodynamic parameters and the estimation of pressure wave} reflections

For each patient, BP was measured three times in the sitting position using a mercury sphygmomanometer, using the first and fifth Korotkoff sounds to identify the systolic and diastolic values, respectively.

Ambulatory BP and arterial distensibility were assessed over $24 \mathrm{~h}(24 \mathrm{~h})$ using a Mobil-O-Graph Arteriograph (I.E.M. GmbH, Stolberg, Germany), an oscillometric device that yields a simultaneous measurement of brachial BP, PWV and AIx. The device is supported with an expert software package, hypertension management system, for the analysis of all registered measurements. Additionally, the device is capable of measuring central BP and AIx using an integrated pulse wave analysis device. Using the Austrian Research Centers Solver method (Austrian Institute of Technology, Vienna, Austria), waveforms were recorded with a regular oscillatory brachial cuff suitable for ambulatory measurement. The Austrian Research Centers Solver method is a novel approach for determining aortic SBP and AIx based on oscillometric BP measurements using a common cuff. The method uses the pulse waves assessed at the brachial artery. ${ }^{25,26}$ The device was applied in the morning, and the patients were allowed to return home with the instruction to attend to their usual activities and return to the hospital the following day for device removal.

Automated results were provided by the device's internal calculations. These results included $24 \mathrm{~h}$, day and night (1) peripheral and central aortic systolic, diastolic and mean BP and pulse pressures; (2) cardiac output (CO); (3) peripheral resistance (PR) (4) nocturnal decreases in systolic BP (NDSBP) and nocturnal decreases in diastolic BP; patients without a nocturnal SBP decline $\geqslant 10 \%$ compared with daytime values were referred to as non-dippers; ${ }^{7}$ (5) aortic augmentation pressure, AIx and AIx standardized against a heart rate of 75 beats per minute (AIx@75); and (6) PWV. Because the accepted cut-off value for AIx with respect to cardiovascular mortality is $25 \%$, we used $25 \%$ as our cut-off value. ${ }^{27}$

\section{Statistical analysis}

Variables are presented either as the mean \pm s.d. or as frequencies. The data had previously been submitted for a normality test (Kolmogorov-Smirnov). In addition to the descriptive statistics (mean \pm s.d. and frequencies), Student's $t$-test was used for normally distributed variables, and the Mann-Whitney $U$-test was used for variables that were not normally distributed. For the comparison of qualitative data, either the chi-square test or Fisher's exact test was used. The correlations among the data were analyzed using Pearson's correlation test. We undertook a linear regression analysis to evaluate the relative significance of the parameters. All tests were two-tailed, and the level of significance was set at $P<0.05$. The data were analyzed using the SPSS statistical package program, version 15, for Windows (SPSS, Chicago, IL, USA).

\section{RESULTS}

Ninety-six patients were enrolled; the majority were female (53\%), with a mean age of $37.98 \pm 11.67$ years; the mean disease duration was $73.75 \pm 88.65$ months; $65.6 \%$ were systolic non-dippers; and $34.4 \%$ exhibited high AIx@75 values. Six (10\%) of the control subjects were systolic non-dippers, and 7 (11.7\%) exhibited high AIx@75. The number of patients with $\mathrm{BD}$ with inactive disease was 53 , whereas 43 patients suffered from active disease. Table 1 summarizes the clinical features, biochemical parameters, BPs and central hemodynamics of the patients with $\mathrm{BD}$ and the control subjects.

Nocturnal SBP decreases correlated with disease duration, albumin, 24-h urinary albumin, central SBP and DBP, 24-h PWV, Night AIx@75 and nocturnal PWV.AIx@75 correlated negatively with height and CRP and correlated positively with $24 \mathrm{~h}$ and nocturnal PR, as well as $24 \mathrm{~h}$ and nocturnal PWV (Table 2). When we performed the multivariate linear regression analysis, NDSBP correlated with 24-h SBP $(\beta=-0.546, P=0.048), 24-\mathrm{h} \operatorname{PWV}(\beta=-3.799, P<0.001)$, nocturnal PWV $(\beta=-3.939, \quad P<0.001), \quad$ nocturnal AIx@75 $(\beta=-0.152, P=0.017) .24-\mathrm{h} \mathrm{PWV}$, nocturnal PWV and nocturnal AIx@75, all of which were significant independent predictors of 
Table 1 Sociodemographic characteristics, biochemical parameters, blood pressures and central hemodynamics in BD patients and controls

\begin{tabular}{|c|c|c|c|}
\hline Parameters & BD patients & Controls & P-values \\
\hline Age (years) & $37.98 \pm 11.67$ & $35.23 \pm 12.58$ & 0.167 \\
\hline Female/male $n(\%)$ & $53(60) / 44(40)$ & $34(57) / 26(43)$ & 0.318 \\
\hline Mean disease duration (months) & $73.75 \pm 88.65$ & - & - \\
\hline Weight (kg) & $69.86 \pm 12.06$ & $67.31 \pm 11.26$ & 0.219 \\
\hline Height $(\mathrm{cm})$ & $1.66 \pm 0.08$ & $1.64 \pm 0.14$ & 0.976 \\
\hline BMI $\left(\mathrm{kg} \mathrm{m}^{-2}\right)$ & $26.56 \pm 4.86$ & $24.51 \pm 5.78$ & 0.176 \\
\hline Glucose (mg dl-1) & $94.20 \pm 20.04$ & $92.43 \pm 7.96$ & 0.698 \\
\hline Total cholesterol (mg dl-1) & $181.67 \pm 43.22$ & $174.79 \pm 30.84$ & 0.384 \\
\hline LDL-cholesterol (mg dl-1) & $114.16 \pm 34.31$ & $121.55 \pm 35.22$ & 0.310 \\
\hline HDL-cholesterol (mg dl-1) & $44.97 \pm 10.48$ & $42.57 \pm 10.45$ & 0.234 \\
\hline Triglycerides $\left(\mathrm{mg} \mathrm{dl}{ }^{-1}\right)$ & $126.46 \pm 76.00$ & $131.81 \pm 43.56$ & 0.685 \\
\hline 24-h SBP & $113.92 \pm 12.57$ & $112.31 \pm 10.34$ & 0.430 \\
\hline 24-h DBP & $73.17 \pm 10.09$ & $71.27 \pm 8.43$ & 0.256 \\
\hline 24-h MBP & $92.44 \pm 14.73$ & $90.15 \pm 8.83$ & 0.303 \\
\hline 24-h pulse pressure & $40.62 \pm 6.20$ & $41.09 \pm 6.61$ & 0.666 \\
\hline 24-h heart rate & $75.88 \pm 9.04$ & $73.00 \pm 10.82$ & 0.089 \\
\hline 24-h Alx@75 & $22.66 \pm 7.94$ & $21.18 \pm 7.45$ & 0.265 \\
\hline Systolic non-dipper $n(\%)$ & 63(65.6) & $6(10)$ & ${ }^{*}<0.001$ \\
\hline The night reduction in SBP & $7.02 \pm 6.79$ & $10.77 \pm 3.30$ & ${ }^{*}<0.001$ \\
\hline Nocturnal SBP & $108.04 \pm 15.45$ & $103.33 \pm 10.29$ & *0.031 \\
\hline Nocturnal DBP & $67.10 \pm 11.96$ & $63.57 \pm 7.90$ & $* 0.040$ \\
\hline Nocturnal MBP & $85.91 \pm 13.10$ & $81.87 \pm 8.05$ & *0.032 \\
\hline Nocturnal pulse pressure & $40.94 \pm 7.33$ & $39.83 \pm 7.53$ & 0.286 \\
\hline Nocturnal heart rate & $67.48 \pm 9.88$ & $66.07 \pm 10.00$ & 0.395 \\
\hline Nocturnal Alx@75 & $21.34 \pm 10.62$ & $19.71 \pm 11.37$ & 0.318 \\
\hline
\end{tabular}

Abbreviations: 24-h, 24-hour; Alx@75, augmentation index value, standardized to a heart rate of 75 beats per minute; BD, Behcet's disease; BMI, body mass index; DBP, diastolic blood pressure; HDL, high-density lipoprotein; LDL, low-density lipoprotein; MAP, mean arterial pressure; SBP, systolic blood pressure.

Values are expressed as mean $+\mathrm{s.d} . * P<0.05$

decreases in nocturnal BP. In the multivariate model, 24h AIx@75 correlated with height $(\beta=-0.338, P=0.040)$, CRP $(\beta=-0.380$, $P=0.004)$ and nocturnal PR $(\beta=0.556, P=0.036)$. The linear regression analysis demonstrated that both height and CRP correlated negatively with increased AIx@75.

The relationships between nocturnal decreases in SBP and the clinical, demographic and laboratory parameters of the patients with $\mathrm{BD}$ were analyzed using $X^{2-}$ and $t$-tests where indicated. Among the patients with non-dipper $\mathrm{BD}$, the AIx values were elevated compared with the dipper patients with BD. Furthermore, the non-dipper patients with $\mathrm{BD}$ exhibited higher night $\mathrm{COs}$ than the dipper patients with BD (Figure 1). The central hemodynamics, BP patterns and arterial stiffness parameters of the non-dipper and dipper patients with $\mathrm{BD}$ are included in Table 3.

When we divided the patients into two groups according to AIx@75 values (the AIx@75<25\% patient group: patients with BD with low AIX vs. AIx@75>25\% patient group: patients with BD with high AIX), the patients with high AIx@75 values were mostly female $(71.0 \%)$ and were shorter $(1.63 \pm 0.08$ vs. $1.68 \pm 0.08 ; P=0.013)$. These patients also exhibited lower high-density lipoprotein $(41.95 \pm 9.76$ vs. $47.93 \pm 9.37 ; P=0.031)$ and creatinine clearance $(125.32 \pm 21.34$ vs. $144.77 \pm 39.16 ; P=0.036)$ values and higher $24 \mathrm{~h}$ PR $(1.35 \pm 0.15$ vs. $1.21 \pm 0.10 ; P<0.001), 24 \mathrm{~h}$ PWV $(5.98 \pm 1.17$ vs. $5.34 \pm 0.70 ; P=0.021)$ and nocturnal $P R(1.38 \pm 0.18$ vs. $1.15 \pm 0.14)$; $P<0.001)$ and PWV $(5.91 \pm 1.26$ vs. $5.20 \pm 0.60 ; P=0.013)$ values than their counterparts in the other group (Figures 2 and 3 ).
Table 2 Correlation coefficients of nocturnal systolic blood pressure decrease and augmentation index value, standardized to a heart rate of 75 beats per minute (Alx@75)

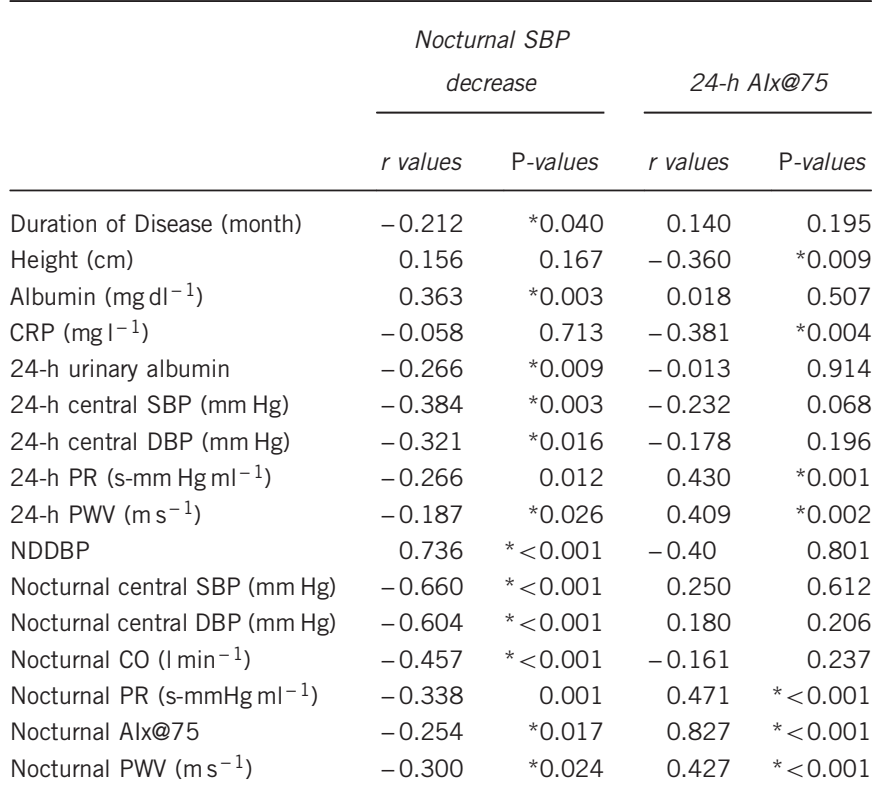

Abbreviations: 24-h, 24-hour. CO, cardiac output. CRP, C-reactive protein; DBP, diastolic blood pressure; NDDBP, nocturnal decrease in the diastolic blood pressure; PR, peripheral resistance; $\mathrm{s}$, second; SBP, systolic blood pressure. ${ }^{*} P<0.05$.

Furthermore, despite the inability to achieve statistical significance, the patients with active BD included a higher percentage of patients with low AIx@75 values (18/58\%vs. 13/41.9\%; $P=0.6)$.

\section{DISCUSSION}

$\mathrm{BD}$ is a chronic relapsing inflammatory process; its predominant histopathology is vasculitis. The vasculitis of BD is distinctive because of the involvement of both veins and arteries of all sizes, although different histological features may be present at different sites. The inflammatory changes in the large arteries are typical of vasculitis, but the lack of granulomatous inflammation and the tendency for aneurysm formation are also distinctive. ${ }^{5,6,28,29}$ This inflammation may contribute to either the initiation or the progression of arterial stiffness. In this study, we evaluated NDSBP, as well as AIx@75, an arterial stiffness parameter, and several additional parameters in patients with BD. In this study, $65.6 \%$ of the patients were systolic non-dippers, whereas $10 \%$ of the control subjects were systolic nondippers. NDSBP correlated with $24 \mathrm{~h}$ PWV, nocturnal CO, nocturnal AIx@75 and nocturnal PWV. AIx@75 correlated with height, CRP and PWV. The patients with high AIx@75 values exhibited higher 24 h PR and PWVs, as well as lower nocturnal COs.

In the present study, $65.6 \%$ of the patients were systolic nondippers, whereas $10 \%$ of control subjects were systolic non-dippers. The incidence of non-dipping BP patterns among the patients with BD patients was similar, and even higher than the incidence of nondipping patterns among the patients with hypertension. Indeed, abnormal decreases in nocturnal BP were observed in $30-50 \%$ of the patients with hypertension. ${ }^{12}$ The non-dippers exhibited higher supine brachial and central SBPs, significantly different central hemodynamics, and elevated left ventricular mass indices compared with the dippers. These cardiovascular anomalies may contribute to an increased risk of cardiovascular mortaility and all-cause mortality. 


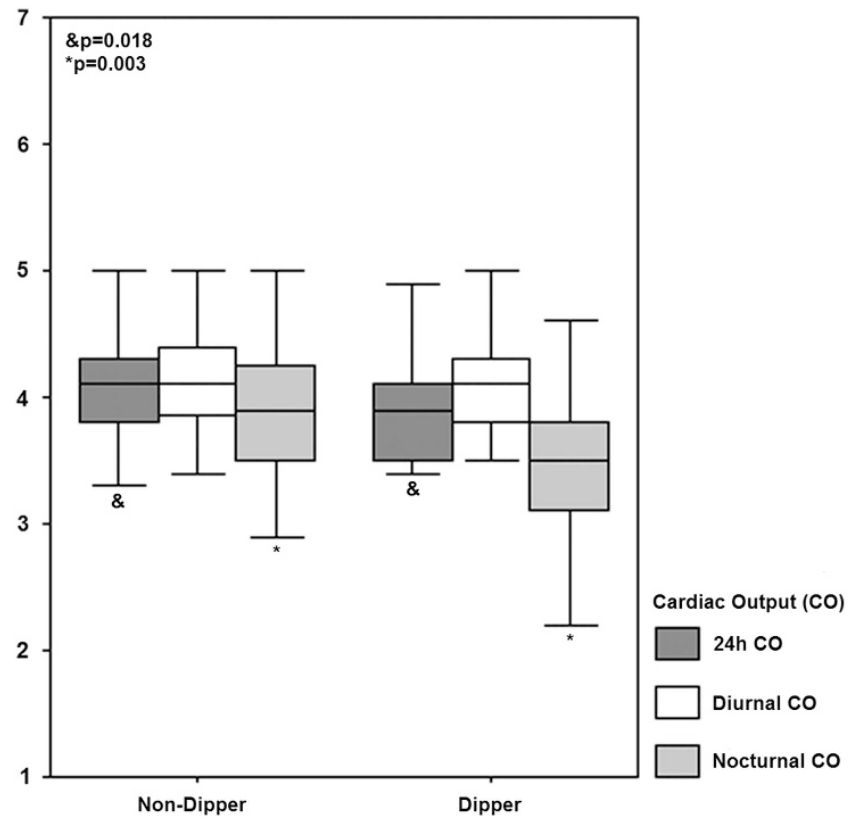

Figure 1 Cardiac output (CO) of dipper and non-dipper patients with Behcet's Disease (BD). Non-dipper BD patients had higher night CO than dipper BD patients.

Many studies have demonstrated that non-dippers suffer more pronounced target organ injury, particularly to the heart, brain and kidney, compared with dippers. ${ }^{11,30}$ In our study, nocturnal SBP decreases correlated with central SBP and DBP, as well as nocturnal decreases in diastolic BP. Additionally, NDSBP correlated negatively with 24-h and nocturnal PWV. The abnormal diurnal central hemodynamic patterns were significant in the patients with $\mathrm{BD}$. The non-dipper patients with BD exhibited higher 24-h central SBPs, 24-h central DBPs, 24-h Alx@75, diurnal minimum SBPs, diurnal minimum DBPs, nocturnal MAPs, pulse pressures, central SBPs, central DBPs, maximum SBPs and maximum DBPs. The deleterious effects of systemic inflammation on the aforementioned surrogate markers of cardiovascular risk may have resulted in increased central BP in the absence of increased peripheral BP and represent a pathophysiological link between inflammation and increased cardiovascular risk. ${ }^{14-17}$ Furthermore, the non-dipper pateints with BD exhibted higher night COs than the dipper patients with $\mathrm{BD}$. Our findings were consistent with those of Takakuwa et al..$^{7}$ and Colleman et al. ${ }^{11}$ who reported that nocturnal cardiac output and stroke volume were significantly higher among non-dippers. The supine brachial SBP abnormality was accompanied by a significantly higher central SBP in the supine position. When the non-dippers moved into the supine position, both the brachial and the central SBP increased, whereas the mean arterial pressure failed to change despite a drop in heart rate. These hemodynamic alterations were initiated by the posture-induced stimulation of cardiopulmonary low-pressure and arterial (carotid and aortic) high-pressure sensors when the non-dippers moved into the supine position. ${ }^{11}$

In this study, 34.4\% of the patients exhibited high AIx@75 values, whereas, $11.7 \%$ of the control subjects exhibited high AIx@75. The percentage of patients with BD with high AIx@75 values was higher than that of the control group. The detrimental effects of systemic inflammation on arterial stiffness are well established. Both the structural and the functional features of the vascular system have
Table 3 The central hemodynamics, BP patterns and arterial stiffness parameters of non-dipper and Dipper BD patients

\begin{tabular}{|c|c|c|c|}
\hline & Non-dipper Patients & Dipper Patients & $\mathrm{P}$ value \\
\hline 24-h SBP (mm Hg) & $115.67 \pm 13.01$ & $109.69 \pm 10.48$ & 0.041 \\
\hline 24-h DBP (mm Hg) & $74.71 \pm 10.31$ & $69.42 \pm 8.63$ & 0.024 \\
\hline 24-h central SBP (mm Hg) & $108.27 \pm 12.45$ & $100.88 \pm 10.08$ & 0.009 \\
\hline 24-h central DBP $(\mathrm{mm} \mathrm{Hg})$ & $76.41 \pm 10.56$ & $70.42 \pm 8.08$ & 0.011 \\
\hline 24-h Alx@75 (\%) & $22.13 \pm 7.60$ & $18.49 \pm 6.42$ & 0.044 \\
\hline 24-h CO $\left(I \mathrm{~min}^{-1}\right)$ & $4.12 \pm 0.42$ & $3.88 \pm 0.37$ & 0.018 \\
\hline DMSBP $(\mathrm{mm} \mathrm{Hg})$ & $94.73 \pm 12.60$ & $88.19 \pm 9.09$ & 0.019 \\
\hline $\mathrm{DMDBP}(\mathrm{mm} \mathrm{Hg})$ & $56.67 \pm 12.19$ & $49.88 \pm 11.11$ & 0.016 \\
\hline $\begin{array}{l}\text { Nocturnal decrease in } \\
\text { DBP }(\mathrm{mm} \mathrm{Hg})\end{array}$ & $8.29 \pm 10.19$ & $20.17 \pm 8.01$ & $<0.001$ \\
\hline Nocturnal SBP (mm Hg) & $112.57 \pm 14.88$ & $97.08 \pm 10.69$ & $<0.001$ \\
\hline Nocturnal DBP $(\mathrm{mm} \mathrm{Hg})$ & $70.57 \pm 11.16$ & $58.69 \pm 9.53$ & $<0.001$ \\
\hline Nocturnal MAP (mm Hg) & $89.76 \pm 12.42$ & $76.58 \pm 9.70$ & $<0.001$ \\
\hline Nocturnal PP (mm Hg) & $41.98 \pm 7.91$ & $38.42 \pm 4.96$ & 0.036 \\
\hline Nocturnal central SBP (mm Hg) & $106.60 \pm 14.56$ & $92.19 \pm 11.06$ & $<0.001$ \\
\hline Nocturnal central DBP $(\mathrm{mm} \mathrm{Hg})$ & $71.65 \pm 11.62$ & $59.85 \pm 9.59$ & $<0.001$ \\
\hline Nocturnal CO $\left(I \mathrm{~min}^{-1}\right)$ & $3.98 \pm 0.63$ & $3.45 \pm 0.60$ & 0.003 \\
\hline Nocturnal Alx@75 (\%) & $20.63 \pm 11.93$ & $17.54 \pm 9.80$ & 0.045 \\
\hline $\begin{array}{l}\text { Nocturnal maximum } \\
\text { SBP }(\mathrm{mm} \mathrm{Hg})\end{array}$ & $129.57 \pm 21.12$ & $115.81 \pm 16.57$ & 0.004 \\
\hline $\begin{array}{l}\text { Nocturnal maximum } \\
\text { DBP }(\mathrm{mm} \mathrm{Hg})\end{array}$ & $84.14 \pm 12.83$ & $74.54 \pm 12.83$ & 0.002 \\
\hline NMSBP $(\mathrm{mm} \mathrm{Hg})$ & $97.98 \pm 14.44$ & $81.65 \pm 10.70$ & $<0.001$ \\
\hline NMDBP $(\mathrm{mm} \mathrm{Hg})$ & $57.16 \pm 12.05$ & $45.19 \pm 8.55$ & $<0.001$ \\
\hline $\operatorname{ESLV}(\%)$ & $16.81 \pm 26.07$ & $5.38 \pm 9.26$ & 0.033 \\
\hline EDLV (\%) & $21.65 \pm 26.95$ & $7.88 \pm 12.88$ & 0.015 \\
\hline
\end{tabular}

Abbreviations: 24-h, 24-hour; Alx@75, augmentation index value, standardized to a heart rate of 75 beats per minute; BD, Behcet's disease; BP, blood pressure; $\mathrm{CO}$, cardiac output; DBP, diastolic blood pressure; DMDBP, diurnal minimum DBP; DMSBP, diurnal minimum SBP; EDLV, exceeding the diastolic limit value; ESLV, exceeding the systolic limit value; MAP, mean arterial pressure: NMDBP, nocturnal minimum DBP; NMSBP; nocturnal minimum SBP; PP, pulse pressure; SBP, systolic blood pressure.

Values are expressed as mean \pm s.d.

become an important issue with respect to $\mathrm{BD},{ }^{16,31}$ which is associated with increased arterial stiffness and involves pressure wave reflections higher than those of control groups. ${ }^{14,15,32-34}$ The gradual increases of arterial stiffness, as well as the decrease in diameter from the central to the peripheral arteries, leads to the generation of pressure wave reflections. ${ }^{32-34}$

In current study, AIx@75 was negatively correlated with CRP. In addition, in despite not reach statistical significance, group of patients with active $\mathrm{BD}$ included higher percentage of patients with low AIx@75 values. The increased arterial stiffness may be attributed to either acute or chronic inflammatory processes associated with BD. ${ }^{17}$ It appears that even relapsing inflammatory episodes may have chronically stable detrimental effects on both the large and the small arteries due to structural changes (for example, arterial remodeling) at the levels of both macro- and the microcirculation. ${ }^{14,16}$ On the other hand, during the acute phase of the inflammatory process, particular patterns of central BPs may develop due to functional changes (peripheral vasodilation), leading to alterations in pressure wave reflections. These deleterious effects of systemic inflammation on the aforementioned surrogate markers of cardiovascular risk may lead to increased central BPs in the absence of increased peripheral BP and represent a pathophysiological link between inflammation and increased cardiovascular risk. ${ }^{14,15}$ Indeed, Vlachopoulos et al. ${ }^{35}$ 


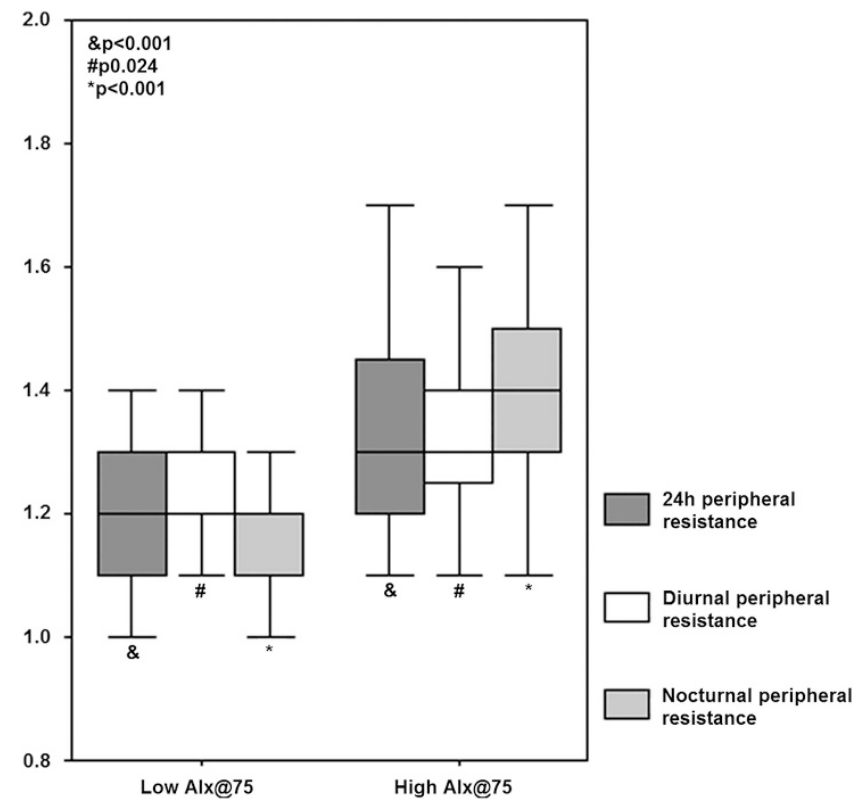

Figure 2 Peripheral resistance (PR) of Behcet's Disease patients with low and high Alx@75. Patients with high Alx@75 had higher 24h PR and nocturnal PR than those of the other group. Alx@75, augmentation index value, standardized to a heart rate of 75 beats per minute.

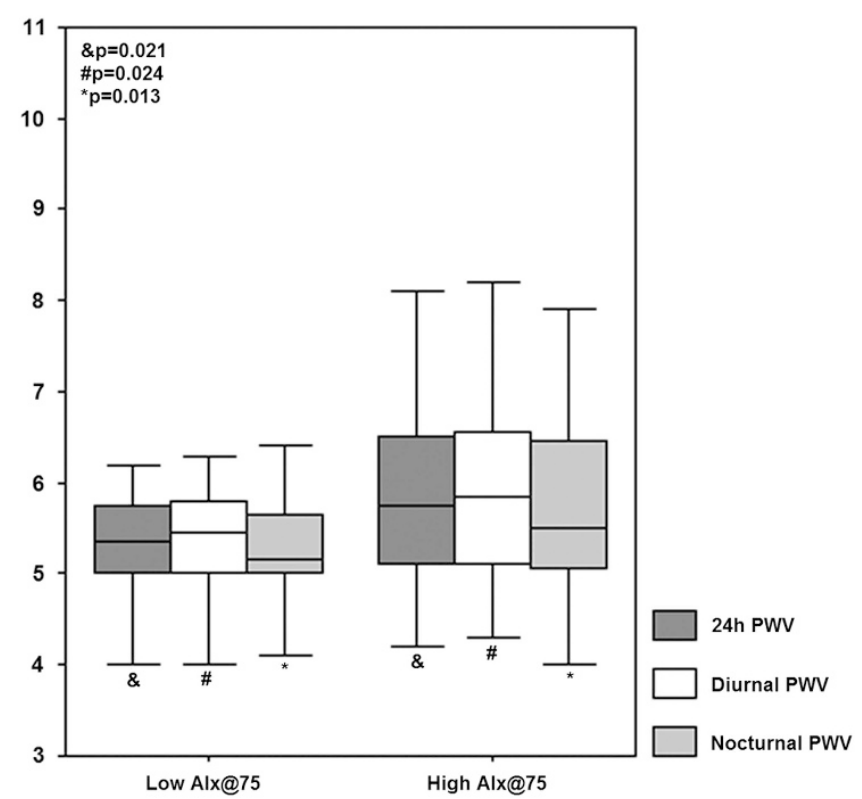

Figure 3 Pulse wave velocity (PWV) of Behcet's Disease patients with low and high Alx@75. Patients with high Alx@75 had higher 24h PWV and nocturnal PWV than those of the other group. Alx@75, augmentation index value, standardized to a heart rate of 75 beats per minute; PWV, Pulse Wave Velocity.

recently demonstrated that although PWV may increase abruptly in the presence of acute inflammation, AIx may decrease. The low-pressure wave reflection indices were attributed to peripheral vasodilation induced by transient acute systemic inflammation, resulting in an attenuation of the reflected pressure wave.
In the present study, AIx@75 correlated negatively with height and correlated positively with $24 \mathrm{~h}$ and nocturnal PR, as well as $24 \mathrm{~h}$ and nocturnal PWV. The patients with high AIx@75 were shorter; the percentage of female patients was higher in the high AIx@75 group. Additionally, the patients with high AIx@75 also exhibited lower nocturnal COs and higher PR and PWV values than the patients with BD patients with low AIx@75. AIx quantifies pressure wave reflections; however, it is dependent not only on the amplitude of the reflected pressure wave but also on the timing of the arrival of the reflected wave within the cardiac cycle. Therefore, AIx also depends on height, sex (due to differences in height), heart rate/ejection duration and PWV. ${ }^{36}$ Although increased arterial stiffness, and hence increased PWV, tends to cause the earlier arrival of the pressure wave, resulting in higher Aix; this effect may be counterbalanced by peripheral vasodilation, tachycardia, or other modulators of the timing of the arrival of the reflected pressure wave under specific conditions. ${ }^{14}$

In conclusion, the present study demonstrated that patients with BD exhibit significantly increased arterial stiffness compared with healthy control subjects. These findings have spawned the hypothesis that the inflammatory processes associated with $\mathrm{BD}$ may cause endothelial dysfunction. Elevations in the markers of endothelial dysfunction (thombomodulin and adhesion molecules) and lowgrade inflammation (proinflammatory cytokines, C-reactive protein and serum amyloid A) are indicative of their roles in the pathophysiology of most of the immune-mediated cardiovascular events associated with $\mathrm{BD} .{ }^{37}$ However, non-dipper systolic hypertension is associated with increased cardiovascular morbidity and mortality. ${ }^{38}$ Patients with systolic non-dipper hypertension were more common in the $\mathrm{BD}$ group than in the control group. There may be a relationship between systolic non-dipper hypertension and BD. Systolic non-dipper status in the setting of $\mathrm{BD}$ may result in subsequent vascular damage within a 24-h period associated with endothelial hyperactivity, which may cause aortic aneurysm. A recent study investigating the relationship between isolated systolic increases in BP (non-dippers) and the incidence of hypertension among elderly patients observed that aortic aneurysms occurred only among non-dippers. ${ }^{39}$ More recently, another study demonstrated that non-dipper patients with hypertension exhibited a higher thoracic aortic diameter compared with dipper patients. ${ }^{40}$ This finding is unusual, as the effect of systolic non-dipper hypertension itself is not believed to be significant among patients with $\mathrm{BD}$; it may associated with the development of aneurysms and nonatherosclerotic coronary artery disease.

\section{Study limitations}

The limitations of our study included the small size of our study population and its high percentage of female patients with $\mathrm{BD}$.

\section{CONCLUSION}

Arterial stiffness and non-dipping status may exacerbate the harmful cardiovascular effects of the other. In addition to conventional risk factors, arterial stiffness and non-dipping status should not be overlooked during the follow-up evaluations of patients with BD. Longitudinal studies in a large population are warranted in order to determine the prognostic implications of increased stiffness parameters in the setting BD. Additionally, future studies with large sample sizes are necessary to elucidate the underlying hemodynamic mechanisms of blunted nocturnal reductions in the BPs of patients with non-dipper BD.

\section{CONFLICT OF INTEREST}

The authors declare no conflict of interest. 
1 Geri G, Wechsler B, Thi Huong du L, Isnard R, Piette JC, Amoura Z, Resche-Rigon M, Cacoub P, Saadoun D. Spectrum of cardiac lesions in Behçet disease: a series of 52 patients and review of the literature. Medicine (Baltimore) 2012; 91: 25-34.

2 Kürüm T, Yildiz M, Soy M, Ozbay G, Alimgil L, Tüzün B. Arterial distensibility as determined by carotid-femoral pulse wave velocity in patients with BD. Clin Rheumatol 2005; 24: 134-138.

3 Marzban M, Mandegar MH, Karimi A, Karimi A, Abbasi K, Movahedi N, Navabi MA, Abbasi SH, Moshtaghi N. Cardiac and great vessel involvement in "BD". J Card Surg 2008; 23: 765-768.

4 Chang JE, Lee YH, Lee J. Multiple cardiovascular complications in a patient with BD. Korean J Intern Med 2008; 23: 100-102.

5 Calamia KT, Schirmer M, Melikoglu M. Major vessel involvement in BD: an update. Cur Opin Rheumatol 2011; 23: 24-31.

6 Melikoglu M, Kural-Seyahi E, Tascilar K, Yazici $\mathrm{H}$. The unique features of vasculitis in Behcet's syndrome. Clin Rev Allergy Immunol 2008; 35: 40-46.

7 Takakuwa H, Ise T, Kato T, Izumiya Y, Shimizu K, Yokoyama H, Kobayashi KI. Diurnal variation of hemodynamic indices in non dipper hypertensive patients. Hypertens Res 2001; 24: 195-201.

8 Brotman DJ, Davidson MB, Boumitri M, Vidt DG. Impaired diurnal blood pressure variation and all-cause mortality. Am J Hypertens 2008; 21: 92-97.

9 Kario K. Proposal of a new strategy for ambulatory blood pressure profile-based management of resistant hypertension in the era of renal denervation. Hypertens Res 2013; 36: 478-484

10 Karpettas N, Destounis A, Kollias A, Nasothimiou E, Moyssakis I, Stergiou GS. Prediction of treatment-induced changes in target-organ damage using changes in clinic, home and ambulatory blood pressure. Hypertens Res 2014; 37: 543-547.

11 Coleman CT, Stowasser M, Jenkins C, Marwick TH, Sharman JE. Central hemodynamics and cardiovascular risk in non- dippers. J Clin Hypertens (Greenwich) 2011; 13: 557-562.

12 Takeda A, Toda T, Fujii T, Matsui N. Bedtime administration of long-acting antihypertensive drugs restores normal nocturnal blood pressure fall in non- dippers with essential hypertension. Clin Exp Nephrol 2009; 13: 467-472.

13 Karpettas N, Nasothimiou E, Kollias A, Vazeou A, Stergiou GS. Ambulatory and home blood pressure monitoring in children and adolescents: diagnosis of hypertension and assessment of target-organ damage. Hypertens Res 2013; 36: 285-292.

14 Ikonomidis I, Lekakis J, Stamatelopoulos K, Markomihelakis N, Kaklamanis PG, Mavrikakis M. Aortic elastic properties and left ventricular function in patients with Adamantiades- BD. J Am Coll Cardiol 2004; 43: 1075-1081.

15 Protogerou AD, Achimastos A, Vlachopoulos C, Stamatelopoulos KS, Papaioannou TG, Papamichael CM, Aznaouridis K, Kaklamanis P, Mavrikakis M, Stefanadis C, Lekakis JP. Reduced pressure wave reflections in patients with active clinical status of Adamantiades-Behcet disease. Hellenic J Cardiol 2008; 49: 408-414.

16 Tunc SE, Dogan A, Gedikli O, Arslan C, Sahin M. Assessment of aortic stiffness and ventricular functions in patients with BD. Rheumatol Int 2005; 25: 447-451.

17 Chang HK, Kim SK, Lee SS, Rhee MY. Arterial stiffness in BD: increased regional pulse wave velocity values. Ann Rheum Dis 2006; 65: 415-416.

18 Mäki-Petäjä KM, Elkhawad M, Cheriyan J, Joshi FR, Ostör AJ, Hall FC, Rudd JH, Wilkinson IB. Anti-Tumor necrosis factor- $\alpha$ therapy reduces aortic inflammation and stiffness in patients with rheumatoid arthritis. Circulation 2012; 126: 2473-2480.

19 Sakuragi S, Abhayaratna WP. Arterial stiffness: methods of measurement, physiologic determinants and prediction of cardiovascular outcomes. Int J Cardiol 2010; 138: $112-118$.

20 Davies JI, Struthers AD. Pulse wave analysis and pulse wave velocity: a critical review of their strengths and weaknesses. J Hypertens 2003; 21: 463-472.
21 Fukui M, Ushigome E, Tanaka M, Hamaguchi M, Tanaka T, Atsuta H, Ohnishi M, Oda Y, Hasegawa G, Nakamura N. Home blood pressure variability on one occasion is a novel factor associated with arterial stiffness in patients with type 2 diabetes. Hypertens Res 2013; 36: 219-225.

22 Mitchell GF, Hwang SJ, Vasan RS, Larson MG, Pencina MJ, Hamburg NM, Vita JA, Levy D, Benjamin EJ. Arterial stiffness and cardiovascular events: the Framingham Heart Study. Circulation 2010; 121: 505-511.

23 Weber T, Auer J, O'Rourke MF, Kvas E, Lassnig E, Berent R, Eber B. Arterial stiffness, wave reflections, and the risk of coronary artery disease. Circulation 2004; 109: 184-189.

24 International Study Group for BD [ISGBD]. Criteria for diagnosis of BD. Lancet 1990; 335: 1078-1080.

25 Wei W, Tölle M, Zidek W, van der Giet M. Validation of the mobil-O-Graph: 24 h-blood pressure measurement device. Blood Press Monit 2010; 15: 225-228.

26 Wassertheurer S, Kropf J, Weber T, van der Giet M, Baulmann J, Ammer M, Hametner B, Mayer CC, Eber B, Magometschnigg D. A new oscillometric method for pulse wave analysis: comparison with a common tonometric method. J Hum Hypertens 2010; 24: 498-504.

27 London GM, Blacher J, Pannier B, Guérin AP, Marchais SJ, Safar ME. Arterial wave reflections and survival in end-stage renal failure. Hypertension 2001; 38: 434-438.

28 Duzgun N, Ates A, Aydintug OT, Demir O, Olmez U. Characteristics of vascular involvement in BD. Scand J Rheumatol 2006; 35: 65-68.

29 O'Duffy JD. Vasculitis in Behçet's disease. Rheum Dis Clin North Am 1990; 16: 423-431.

30 Routledge FS, McFetridge-Durdle JA, Dean CR. Night-time blood pressure patterns and target organ damage: a review. Can J Cardiol 2007; 23: 132-138.

31 Protogerou A, Lekakis J, Stamatelopoulos K, Aznaouridis K, Karatzis E, Ikonomidis I, Papamichael Ch, Markomihelakis N, Mavrikakis M, Kaklamanis PG. Arterial wall characteristics in patients with Adamantiades-Behcet'sdisease. Adv Exp Med Biol 2003; 528: 399-404.

32 Rhee MY, Chang HK, Kim SK. Intima-media thickness and arterial stiffness of carotid artery in Korean patients with BD. J Korean Med Sci 2007; 22: 387-392.

33 Chambers JC, Haskard DO, Kooner JS. Vascular endothelial function and oxidative stress mechanisms in patients with Behçet's syndrome. J Am Coll Cardiol 2001; 37: 517-520.

34 Booth AD, Wallace S, McEniery CM, Brown J, Jayne DR, Wilkinson IB. Inflammation and arterial stiffness in systemic vasculitis. A model of vascular inflammation. Arthritis Rheum 2004; 50: 581-588.

35 Vlachopoulos C, Dima I, Aznaouridis K, Vasiliadou C, loakeimidis N, Aggeli C, Toutouza $\mathrm{M}$, Stefanadis $\mathrm{C}$. Acute systemic inflammation increases arterial stiffness and decreases wave reflections in healthy individuals. Circulation 2005; 112: 2193-2200.

36 Mitchell G. Triangulating the peaks of arterial stiffness. Hypertension 2006; 48 : 543-545.

37 Van Bussel BC, Schouten F, Henry RM, Schalkwijk CG, de Boer MR, Ferreira I, Smulders YM, Twisk JW, Stehouwer CD. Endothelial dysfunction and low-grade inflammation are associated with greater arterial stiffness over a 6-year period. Hypertension 2011; 58: 588-595.

38 Ohkubo T, Hozawa A, Yamaguchi J, Kikuya M, Ohmori K, Michimata M, Matsubara M, Hashimoto J, Hoshi H, Araki T, Tsuji I, Satoh H, Hisamichi S, Imai Y. Prognostic significance of the nocturnal decline in blood pressure in individuals with and without high 24-h blood pressure: the Ohasama study. J Hypertens 2002; 20: 2183-2189.

39 Zain-EI MH, Snincak M, Pahuli K, Solarova Z, Hrabcakova P. Non-dipping morning blood pressure and isolated systolic hypertension in elderly. Bratis/ Lek Listy 2013; 114: 150-154.

40 Karaagac K, Vatansever F, Tenekecioglu E, Ozluk OA, Kuzeytemiz M, Topal D, Yilmaz $M$. The relationship between non-dipper blood pressure and thoracic aortic diameter in metabolic syndrome. Eurasian J Med 2014; 46: 120-125. 